\title{
The prevalence of pulmonary embolism in non-hospitalised de-isolated patients diagnosed with mild COVID-19 disease
}

\author{
O Evbuomwan, ${ }^{1}$ MBBS, FNCP (SA), MMed (Nucl Med); G Engelbrecht, ${ }^{1}$ MB BCh, FNCP (SA), MMed (Nucl Med); \\ M V Bergman, ${ }^{2} \mathrm{MB}$ BCh; S Mokwena, ${ }^{2} \mathrm{MB}$ BCh; O A Ayeni, ${ }^{3} \mathrm{MB} \mathrm{ChB}, \mathrm{MSc}, \mathrm{PhD}$ \\ ${ }^{1}$ Department of Nuclear Medicine, Faculty of Health Sciences, University of the Free State and Universitas Academic Hospital, Bloemfontein, \\ South Africa \\ ${ }^{2}$ Occupational Health Unit, Universitas Academic Hospital, Bloemfontein, South Africa \\ ${ }^{3}$ Non-Communicable Diseases Research Division, Wits Health Consortium, University of the Witwatersrand, Johannesburg, South Africa
}

Corresponding author: O Evbuomwan(moreli14@yahoo.com)

\begin{abstract}
Background. Pulmonary embolism (PE) is a known complication of COVID-19 disease. The mechanism of thromboembolic events appears to be stimulated by excessive thrombin production, inhibition of fibrinolysis and deposition of antiphospholipids and thrombi, as well as microvascular dysfunction in multiple vascular beds. The occurrence of PE has been well demonstrated in hospitalised patients with severe disease. Very few data are available on its incidence or prevalence in non-hospitalised patients diagnosed with a milder form of the disease. Objectives. To assess the prevalence of PE in non-hospitalised patients diagnosed with mild COVID-19 who presented with raised D-dimer levels and persistent or new-onset cardiopulmonary symptoms.

Methods. This was a retrospective study conducted in the Department of Nuclear Medicine at Universitas Academic Hospital, Bloemfontein, South Africa. We reviewed the studies of 65 non-hospitalised patients with COVID-19 referred to the department from July 2020 to January 2021 for a perfusion-only single-photon emission computed tomography/computed tomography (SPECT/CT) study or a ventilation/ perfusion (VQ) SPECT/CT study. All 65 patients had raised D-dimer levels with persistent, worsening or new-onset cardiopulmonary symptoms after the diagnosis of COVID-19.

Results. Sixty-five patients were studied. The median (interquartile range) age was 46 (41 - 54) years and the majority (88.2\%) were female. There were 22 patients (33.8\%) with lung perfusion defects in keeping with PE. Two of these patients had a false-negative computed tomography pulmonary angiography (CTPA) study for PE performed the same day as their VQ SPECT/CT study.

Conclusions. We confirm a high prevalence of PE in non-hospitalised patients diagnosed with mild COVID-19 who presented with raised D-dimer levels and persistent or new-onset cardiopulmonary symptoms. We recommend that irrespective of disease severity, hospitalised and non-hospitalised patients with COVID-19 presenting with persistent or new-onset cardiopulmonary symptoms and raised D-dimer levels should be investigated further for PE.
\end{abstract}

S Afr Med J 2021;111(8):741-746. https://doi.org/10.7196/SAMJ.2021.v111i8.15657

COVID-19 disease results in a wide spectrum of clinical manifestations including flu-like symptoms, difficulty in breathing, blood and circulatory complications, gastrointestinal symptoms, hepatocellular injury, hyperglycaemia and ketosis, neurological illnesses,ocular symptoms and dermatological complications. ${ }^{[1]}$ These effects are thought to occur as a result of angiotensin-converting enzyme 2-mediated cellular viral entry, tissue damage, dysregulation of the renin-angiotensin-aldosterone system, systemic release of cytokines, and dysfunctions in the microcirculation. ${ }^{[2,3]}$

Several reports have documented an increased incidence of venous thromboembolic events, including pulmonary embolism (PE), among critically ill patients with COVID-19 admitted to hospitals, including intensive care units (ICUs). ${ }^{[2,4-11]}$ The mechanism of thromboembolic events seems to be stimulated by excessive thrombin production, inhibition of fibrinolysis and deposition of antiphospholipids and thrombi, as well as microvascular dysfunction in multiple vascular beds including the lungs, brain, kidneys and heart. ${ }^{[1]}$ COVID-19 is also known to predispose patients to systemic inflammation, which has been reported to increase the risk of deep-vein thrombosis, with PE seen in 16.7 - 47\% of patients admitted to ICUs. ${ }^{[2]}$

Little is known about the incidence or prevalence of PE in nonhospitalised patients diagnosed with a milder form of the disease.
Anecdotal evidence during the early period of the pandemic at our facility, the Department of Nuclear Medicine at Universitas Academic Hospital in Bloemfontein, South Africa (SA), suggested that some non-hospitalised patients diagnosed with mild COVID-19 were presenting later with persistent or new-onset cardiopulmonary symptoms after de-isolation. Ventilation/perfusion (VQ) singlephoton emission computed tomography/computed tomography (SPECT/CT) studies performed in those with raised D-dimer levels revealed perfusion defects in keeping with PE.

\section{Objectives}

To assess the prevalence of PE in this cohort of patients with raised D-dimer levels.

\section{Methods}

Ethics approval was obtained from the Health Sciences Research Ethics Committee at the University of the Free State (ref. no. UFSHSD2020/1544/2411).

\section{Study design and location}

The study was a retrospective cohort study conducted in a tertiary institution. 


\section{Study population}

We reviewed all the perfusion-only SPECT/CT and VQ SPECT/CT studies of 184 patients who were investigated for PE between July 2020 and January 2021. Sixty-five patients who met the inclusion criteria were included in the study. All had raised D-dimer levels and persistent or new-onset cardiopulmonary symptoms after de-isolation. None had been hospitalised for severe disease. Forty-seven patients had a perfusion-only SPECT/CT study as their initial study, while 18 had a VQ SPECT/CT study. Perfusion-only studies were performed because earlier on in the pandemic we were being cautious with ventilating patients owing to potential spread of infection. It is usually routine practice in our facility for patients with $\mathrm{PE}$ to have a repeat study 3 months after initiating therapeutic anticoagulation. However, only 12 patients came for a follow-up VQ SPECT/CT study 3 months after initiation of therapeutic anticoagulation for PE.

\section{Inclusion criteria}

- Non-hospitalised de-isolated patients diagnosed with mild COVID-19 infection

- Age $\geq 18$ years

- Raised D-dimers

- Persistent or new-onset cardiopulmonary symptoms after de-isolation.

\section{Exclusion criteria}

- All patients without a diagnosis of COVID-19

- Patients diagnosed with severe COVID-19

- All hospitalised patients diagnosed with COVID-19.

\section{Equipment}

Ventilation studies were performed with 20 - 25 mCi of technetium-99 metastable diethylenetriamine pentacetate, using the SmartVent radioaerosol delivery system (Diagnostic Imaging Ltd, UK). Perfusion studies were performed with $3-5 \mathrm{mCi}$ of technetium-99 macroaggregated albumin.

Images were acquired with either a 16-slice SPECT/CT camera (Siemens Symbia T16 TruePoint; Siemens Medical Solutions USA, Inc.) or a 2-slice SPECT/CT camera (Siemens Symbia T2 TruePoint; Siemens Medical Solutions USA, Inc.). Both cameras are dual-headed gamma cameras, with similar work stations and processing units.

\section{Acquisition protocol}

Both gamma cameras were equipped with a low-energy highresolution collimator. For those patients who had ventilation studies done, SPECT imaging was acquired immediately after ventilation of the radioaerosol at $15 \mathrm{~s} / \mathrm{stop}$, with $3^{\circ}$ steps, in a $128 \times 128$ matrix. Then perfusion SPECT imaging was acquired after injection of the perfusion tracer at $12 \mathrm{~s} / \mathrm{stop}$, with $3^{\circ}$ steps, in a $128 \times 128$ matrix. This was followed by a low-dose non-contrast chest computed tomography scan, with the patient remaining in the same position.

\section{Image processing}

Images were processed using the Syngo work stations for both gamma cameras. SPECT images were reconstructed using an iterative algorithm, and SPECT/CT fusion images were obtained using the multimodality Syngo imaging software on the workstation.

\section{Image interpretation}

Two nuclear medicine physicians with combined experience of 36 years (27 and 9 years) interpreted the images. Interpretation of the VQ SPECT/CT studies was based on the accepted European guidelines, using at least one large segmental or two subsegmental unmatched perfusion defects for the diagnosis of PE. ${ }^{[12]}$ Interpretation of the perfusion-only SPECT/CT study was based on a modification of the Prospective Investigative Study of Acute Pulmonary Embolism Diagnosis study, using at least one large wedge-shaped segmental perfusion defect, with normal or near-normal CT findings in that segment for the diagnosis of PE. ${ }^{[13,14]}$ These findings were not compared with a gold standard, as there is no available modality alone good enough to serve as a gold standard for the diagnosis of PE. However, for those patients who had follow-up studies, an improvement in the size of the perfusion defects and symptomatic improvement 3 months after initiating anticoagulation was used as a confirmation for PE.

\section{Data analysis}

Data from each patient were collected using Excel 2019 (Microsoft, USA) and analysed using the statistical package Stata version 16 (StataCorp, USA).

\section{Statistical analysis}

We reported frequencies and percentages for categorical variables, and medians and interquartile ranges (IQRs) for continuous variables. Associations of age, time between COVID-19 infection and the scan, symptoms prompting the scan, D-dimer levels and COVID pneumonia with $\mathrm{PE}$ were evaluated using the Wilcoxon rank-sum test for comparison of continuous variables and Pearson's $\chi^{2}$ test for categorical variables. We used binary logistic regression to determine the effect size of associations of these covariates with PE, reporting odds ratios (ORs) and 95\% confidence intervals (CIs). A two-tailed $p$-value $<0.05$ was considered statistically significant.

\section{Results}

Sixty-five patients were enrolled during the study period between July 2020 and January 2021. The median (IQR) age was 46 (41 - 54) years, and the majority (89.2\%) were female (Table 1$)$. All patients had raised D-dimer levels and new-onset or persistence of cardiopulmonary symptoms. The most common symptom was shortness of breath (70.8\%), followed by chest pain (41.5\%) and tachycardia (21.5\%) (Fig. 1).

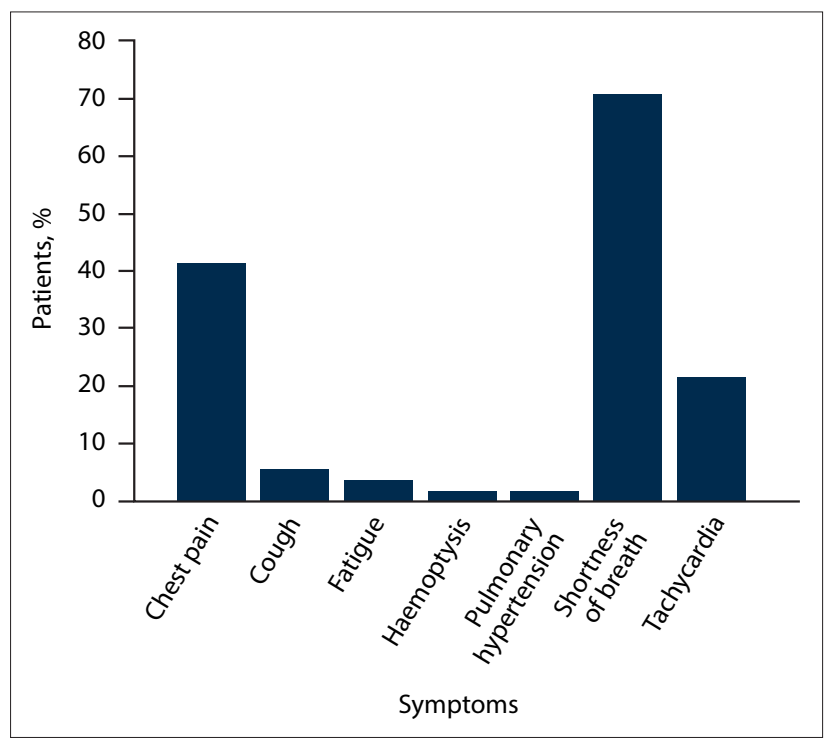

Fig. 1. Distribution of symptoms prompting referral for the study. 
Table 1. Demographic and clinical characteristics of the patients $(N=65)$

\begin{tabular}{ll}
\hline Characteristics & \\
\hline Age (years), median (IQR) & $46(41-54)$ \\
Sex, $n$ (\%) & $58(89.2)$ \\
$\quad$ Female & $7(10.8)$ \\
Male & $15(14-25)$ \\
Time between COVID-19 infection and scan (days), median (IQR) & $0.34(0.28-0.81)$ \\
D-dimers (mg/L), median (IQR) & \\
Pulmonary embolism, $n$ (\%) & $43(66.2)$ \\
$\quad$ No & $22(33.8)$ \\
Yes & \\
COVID lungs, $n(\%)$ & $39(60.0)$ \\
$\quad$ No & $26(40.0)$ \\
Yes & \\
Follow-up study, $n$ (\%) & $42(75.0)$ \\
None & $14(25.0)$ \\
VQ SPECT/CT scan &
\end{tabular}

Table 2. Associations of SPECT/CT findings with pulmonary embolism

\begin{tabular}{|c|c|c|c|c|}
\hline & \multicolumn{2}{|c|}{ Pulmonary embolism } & \multirow[b]{2}{*}{ OR $(95 \% \mathrm{CI})$} & \multirow[b]{2}{*}{$p$-value } \\
\hline & No $(N=43)$ & Yes $(N=22)$ & & \\
\hline Age (years), median (IQR) & $45(39-54)$ & $48(43-54)$ & $1.04(0.98-1.10)$ & 0.267 \\
\hline $\begin{array}{l}\text { Interval between COVID-19 infection and VQ scan (days), } \\
\text { median (IQR) }\end{array}$ & $15(14-25)$ & $15(12-25)$ & $1.02(0.98-1.05)$ & 0.796 \\
\hline \multicolumn{5}{|l|}{ Symptoms prompting VQ scan, $n(\%)$} \\
\hline Shortness of breath & & & & $0.249^{*}$ \\
\hline No & $15(34.9)$ & $4(18.2)$ & 1.00 (reference) & \\
\hline Yes & $28(65.1)$ & $18(81.8)$ & $2.41(0.69-8.43)$ & \\
\hline Tachycardia & & & & 0.868 \\
\hline No & $34(79.1)$ & $17(77.3)$ & 1.00 (reference) & \\
\hline Yes & $9(20.9)$ & $5(22.7)$ & $1.38(0.37-5.10)$ & \\
\hline Chest pain & & & & 0.545 \\
\hline No & $24(55.8)$ & $14(63.6)$ & 1.00 (reference) & \\
\hline Yes & $19(44.2)$ & $8(36.4)$ & $0.72(0.25-2.08)$ & \\
\hline Cough & & & & 0.999 \\
\hline No & $41(95.3)$ & $21(95.5)$ & 1.00 (reference) & \\
\hline Yes & $2(4.7)$ & $1(4.5)$ & $0.98(0.08-11.40)$ & \\
\hline Haemoptysis & & & & $0.545^{*}$ \\
\hline No & $41(95.3)$ & $22(100.0)$ & 1.00 (reference) & \\
\hline Yes & $2(4.7)$ & $0(0.0)$ & Omitted & \\
\hline Pulmonary hypertension & & & & $0.999^{*}$ \\
\hline No & $42(97.7)$ & $22(100.0)$ & 1.00 (reference) & \\
\hline Yes & $1(2.3)$ & $0(0.0)$ & Omitted & \\
\hline Fatigue & & & & $0.111^{*}$ \\
\hline No & $43(100.0)$ & $20(90.9)$ & 1.00 (reference) & \\
\hline Yes & $0(0.0)$ & $2(9.1)$ & Omitted & \\
\hline D-dimers (mg/L), median (IQR) & $0.31(0.26-0.74)$ & $0.41(0.31-0.87)$ & $1.07(0.54-2.09)$ & 0.061 \\
\hline COVID pneumonia, $n(\%)$ & & & & 0.087 \\
\hline No & $29(67.4)$ & $10(45.5)$ & 1.00 (reference) & \\
\hline Yes & $14(32.6)$ & $12(54.5)$ & $2.49(0.87-7.13)$ & \\
\hline
\end{tabular}


All the patients had lung perfusion studies performed after de-isolation, 10 - 90 days after the diagnosis of COVID-19 infection, with $47(72.3 \%)$ of them having a perfusiononly SPECT/CT study, while only 18 (27.7\%) had a VQ SPECT/CT study. Of these 65 patients, $12(18.5 \%)$ had a follow-up VQ SPECT/CT study 3 months after their initial study. These follow-up studies showed either partial or complete resolution of the perfusion defects seen earlier.

There were 22 patients (33.8\%) with lung perfusion defects in keeping with PE. Fourteen (63.6\%) of the 22 patients with PE had one large single segmental perfusion defect, 6 (27.3\%) had two large segmental perfusion defects, while only $2(9.1 \%)$ had three large segmental perfusion defects.

The median (IQR) age of the patients with PE was not significantly different from that of the patients without $(48(43-54)$ v. 45 (39 - 54) years, respectively; $p=0.267$ ), and the difference between the median (IQR) D-dimer levels in patients with and without $\mathrm{PE}$ was of marginal significance $(0.41(0.31-0.87) \mathrm{mg} / \mathrm{L}$ v. $0.31(0.26-0.74) \mathrm{mg} / \mathrm{L} ; p=0.254)$. For every 0.1 unit increase in D-dimer level, patients had a $7 \%$ increased odds of presenting with $\mathrm{PE}$ (OR 1.07; 95\% CI $0.54-2.09 ; p=0.060$ ). Those who had COVID pneumonia were more likely to have PE. However, this was of marginal significance (OR 2.49; 95\% CI 0.87 - 7.13; $p=0.087$ ) (Table 2).

\section{Discussion}

This study shows that of non-hospitalised patients with raised $\mathrm{D}$-dimers and persistent or new-onset cardiopulmonary symptoms diagnosed with mild COVID-19 disease, about a third were diagnosed with $\mathrm{PE}$ on perfusion-only or VQ SPECT/CT studies.

$\mathrm{PE}$ is a known thrombotic complication that has been associated with COVID-19 infection. ${ }^{[6]}$ Numerous studies have shown the prevalence of $\mathrm{PE}$ in hospitalised patients with severe disease, ${ }^{[2,4-11]}$ but there are not yet sufficient data on patients like ours, with persistent cardiopulmonary symptoms after recovery from mild disease. However, some case reports have reported the occurrence of $\mathrm{PE}$ in non-hospitalised patients diagnosed with COVID-19 infection. ${ }^{[15-18]}$

In our study, there were 22 patients (33.8\%) with lung perfusion defects in keeping with PE (Figs 2 and 3). We suspect that the significant number of cases with $\mathrm{PE}$ in our cohort was due to the peculiar characteristics of our study population (raised D-dimer levels and persistent or new-onset cardiopulmonary symptoms). If we were to include all non-hospitalised patients diagnosed with mild COVID-19

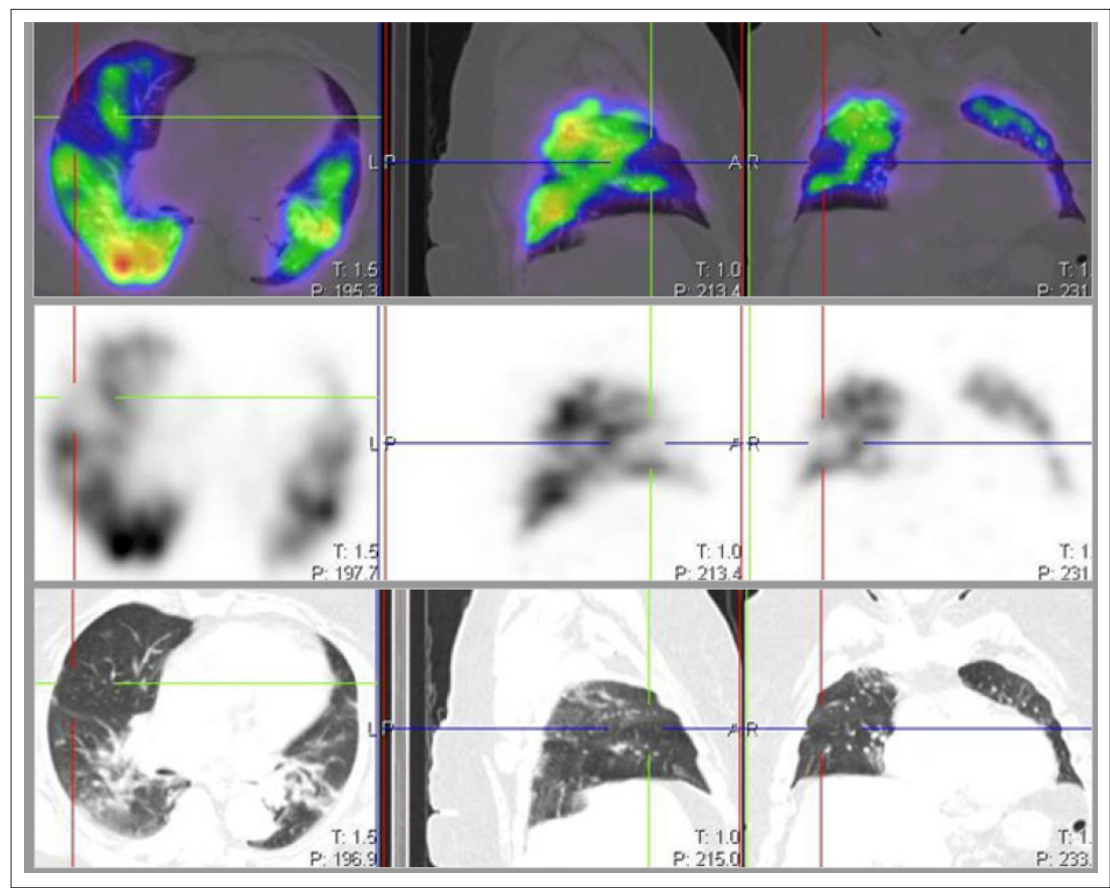

Fig. 2. Perfusion single-photon emission computed tomography/computed tomography images showing a perfusion defect (crosshair) in the lateral segment of the right middle lobe prior to anticoagulation therapy.

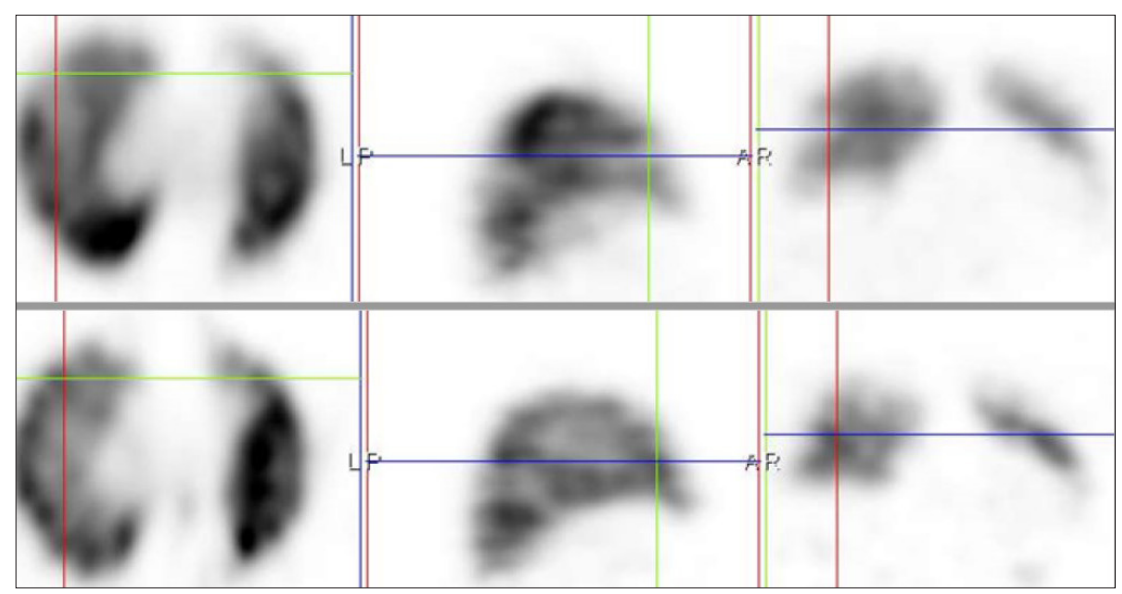

Fig. 3. Ventilation/perfusion single-photon emission computed tomography/computed tomography images showing some resolution of the defect seen in Fig. 2, 3 months after initiating anticoagulation. The bottom images are the ventilation images confirming a mismatch of the perfusion defect.

disease, we would probably have a lower percentage of patients with PE. Twelve out of the 22 patients with PE had follow-up VQ SPECT/CT studies 3 months after initiation of therapeutic anticoagulation. These followup studies showed some form of defect resolution ranging from partial to complete, which corresponded with symptomatic improvement (Fig. 3).

Many of the studies that have looked at the incidence of PE in COVID-19 infection used CTPA as the diagnostic modality. However, CTPA has limitations in the diagnosis of chronic PE and detection of peripheral emboli, for which it has low sensitivity. ${ }^{[12,19]}$ In our study, we had two cases of falsenegative CTPA studies, with a positive VQ
SPECT/CT study performed the same day (Fig. 4). These patients both presented with persistent cardiopulmonary symptoms 3 months after the diagnosis of COVID-19 infection and were probably cases of chronic PE. It is therefore likely that CTPA may miss most of the chronic presentations and cases with peripheral emboli.

Hospitalised patients with COVID-19 infection are likely to receive therapeutic or prophylactic anticoagulation and to have investigations performed to diagnose PE. This is not the case for non-hospitalised patients, in whom there is a high chance of PE going unnoticed, which could predispose them to future complications such as pulmonary hypertension and/or 


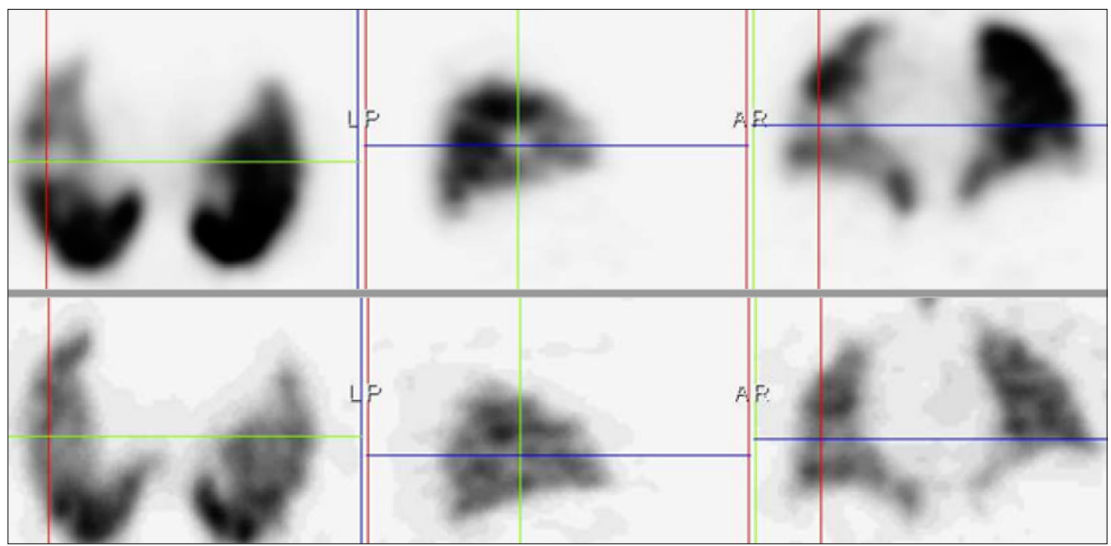

Fig. 4. Ventilation/perfusion single-photon emission computed tomography/computed tomography images confirming an unmatched large perfusion defect in the lateral segment of the right middle lobe in a patient with persistent cardiopulmonary symptoms 3 months after the diagnosis of COVID. A computed tomography pulmonary angiography study done earlier the same day was negative for pulmonary embolism.

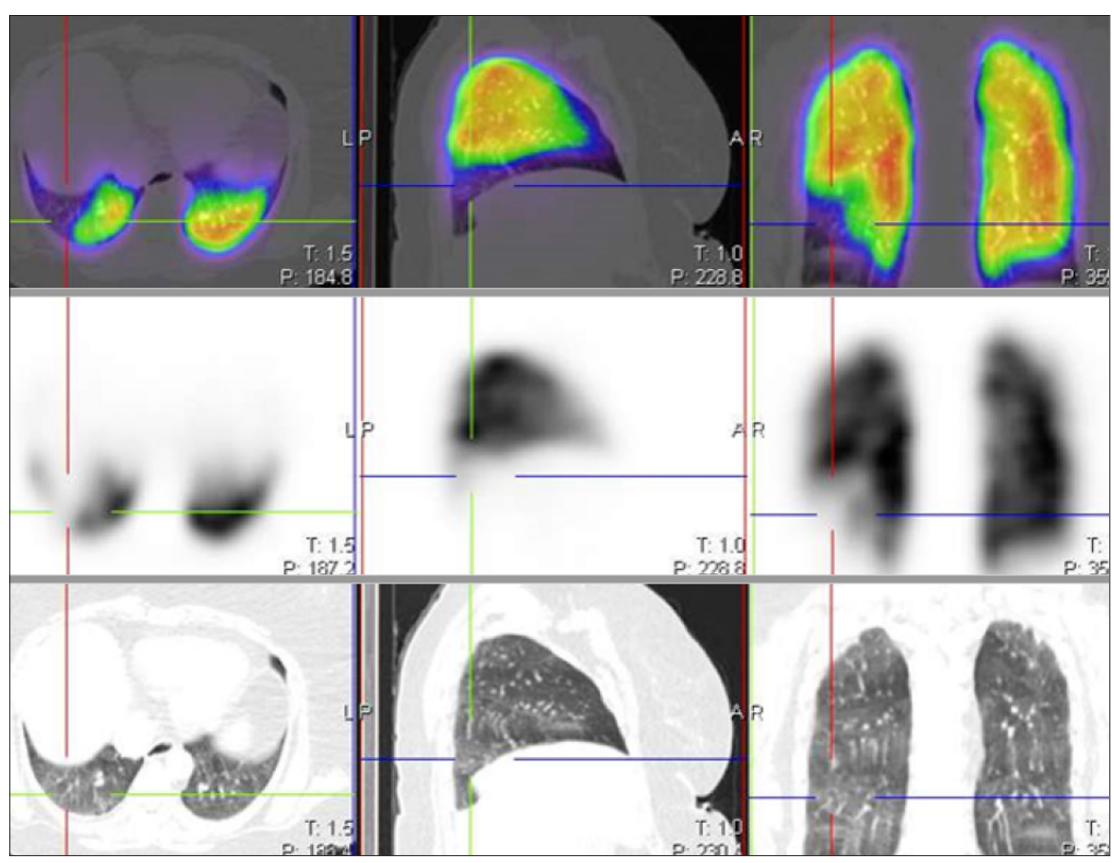

Fig. 5. Perfusion single-photon emission computed tomography/computed tomography images showing large perfusion defects in the posterior and lateral basal segments of the right lower lobe in a patient on prophylactic anticoagulation.

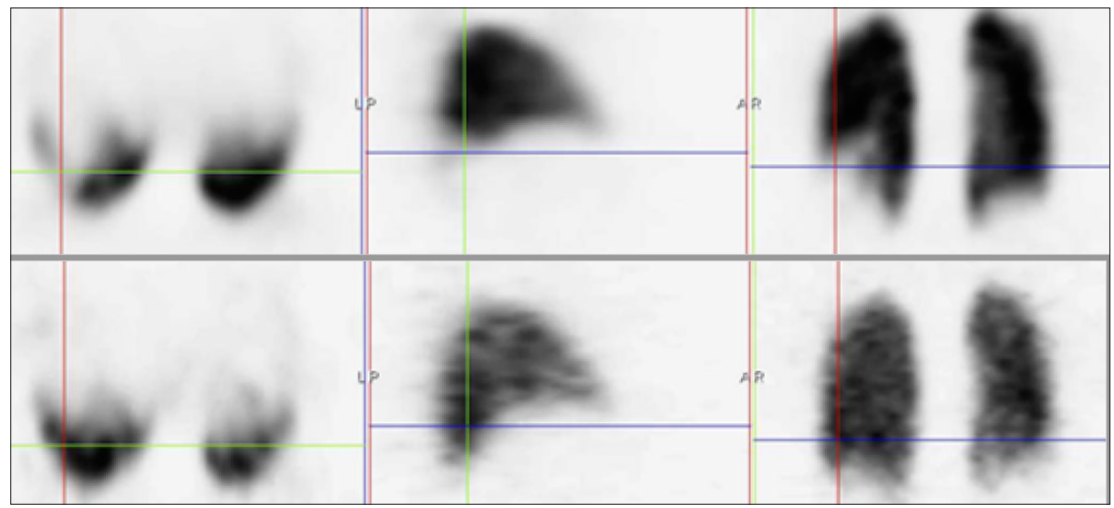

Fig. 6. Ventilation/perfusion single-photon emission computed tomography/computed tomography images demonstrating normal ventilation (bottom images) in the same case as in Fig. 5, thus confirming pulmonary embolism. right heart failure. In addition, developing a second PE could be fatal in this category of patients, especially if the first episode was not diagnosed and treated.

Reports in the literature have suggested that even with prophylactic anticoagulation, the incidence of thrombotic complications in patients with COVID-19 infection is high. ${ }^{[8,10]}$ One of the patients in our cohort was actually on anticoagulation prophylaxis with a novel oral anticoagulant, starting on the day of diagnosis of COVID-19 infection and 2 days after symptom onset (fever, headache and body pains). She developed chest pain shortly before de-isolation. This pain was persistent for about 8 weeks before she reported for a VQ study, which was positive for PE (Figs 5 and 6).

We believe that going forward, even after the end of this pandemic, there may be a rise in the incidence of pulmonary hypertension and right heart failure from undetected and untreated $\mathrm{PE}$ in non-hospitalised patients with COVID-19 infection. In our facility, we are already beginning to see an increase in referrals for VQ studies to diagnose PE in patients with unexplained pulmonary hypertension. It is also likely that some of the cardiopulmonary symptoms being associated with 'long' COVID may in fact be due to undetected and untreated PE.

\section{Study limitations}

Some limitations have been observed in this study, which may contribute to the high prevalence of PE. First, there is a possibility of increased false-positive rates of PE when perfusion-only studies are performed without ventilation. Second, we do not have an accurate pretest probability for all the patients, and we have not been able to rule out other risk factors for PE in these patients. Third, we are not certain whether the referral doctors correctly diagnosed all these patients as having mild COVID-19 infection, and there is a possibility that some of them may have had moderate disease. We therefore recommend that a proper prospective multicentre study be carried out in this patient population in SA, as this will show the true incidence of PE in nonhospitalised patients diagnosed with mild COVID-19 infection.

\section{Conclusions}

$\mathrm{PE}$ is not an uncommon occurrence in a certain category of non-hospitalised patients diagnosed with mild COVID-19 infection who present with raised D-dimer levels and persistent or new-onset cardiopulmonary symptoms. This information is important for the scientific community and healthcare 
workers. We recommend that irrespective of the severity of COVID19 infection, hospitalised and non-hospitalised patients with raised D-dimer levels and persistent or new-onset cardiopulmonary symptoms be investigated for PE with a modality that can detect both acute and chronic cases.

\section{Declaration. None.}

Acknowledgements. With their permission, the authors acknowledge all the radiographers, nurses and other staff in the Department of Nuclear Medicine at Universitas Academic Hospital.

Author contributions. OE was the major contributor in writing the article, GE was a contributor in writing and proofreading the article, MVB and SM played major roles in data collection, and OAA analysed and interpreted the data. All authors read and approved the final manuscript and have given consent for publication of this article.

Funding. None.

Conflicts of interest. None.

1. Gupta A, Madhavan MV, Sehgal K, et al. Extrapulmonary manifestations of COVID-19. Nat Med 2020;26:1017-1032. https://doi.org/10.1038/s41591-020-0968-3

2. Whyte MB, Kelly PA, Gonzalez E, Arya R, Roberts LN. Pulmonary embolism in hospitalised patients Whyte MB, Kelly PA, Gonzalez E, Arya R, Roberts LN. Pulmonary embolism in hospitalised pa
with COVID-19. Thromb Res 2020;195:95-99. https://doi.org/10.1016/j.thromres.2020.07.025

3. Connors JM, Levy JH. Thromboinflammation and the hypercoagulability of COVID-19. J Thromb Haemost 2020;18(7):1559-1561. https://doi.org/10.1111/jth.14849

4. Klok FA, Kruip MJHA, van der Meer NJM, et al. Confirmation of the high cumulative incidence of thrombotic complications in critically ill ICU patients with COVID-19: An updated analysis. Thromb Res 2020;191:148-150. https://doi.org/10.1016/j.thromres.2020.04.041

5. Faggiano P, Bonelli A, Paris S, et al. Acute pulmonary embolism in COVID-19 disease: Preliminary report on seven patients. Int J Cardiol 2020;313:129-131. https://doi.org/10.1016/j.ijcard.2020.04.028 6. Poyiadji N, Cormier P, Patel PY, et al. Acute pulmonary embolism and COVID-19. Radiology 2020;297(3):335-338. https://doi.org/10.1148/radiol.2020201955
7. Klok FA, Kruip MJHA, van der Meer NJM, et al. Confirmation of the high cumulative incidence of thrombotic complications in critically ill ICU patients with COVID-19: An updated analysis. Thromb Res 2020;191:148-150. https://doi.org/10.1016/j.thromres.2020.04.041

8. Klok FA, Kruip MJHA, van der Meer NJM, et al. Incidence of thrombotic complications in critically ill ICU patients with COVID-19. Thromb Res 2020;191:145-147. https://doi.org/10.1016/j. hromres.2020.04.01

9. Grillet F, Behr J, Calame P, Aubry S, Delabrousse E. Acute pulmonary embolism associated with COVID-19 pneumonia detected with pulmonary CT angiography. Radiology 2020;296(3):186-188. https://doi.org/10.1148/radiol.2020201544

10. Lodigiani C, Iapichino G, Carenzo L, et al. Venous and arterial thromboembolic complications in COVID-19 patients admitted to an academic hospital in Milan, Italy. Thromb Res 2020;191:9-14. https://doi.org/10.1016/j.thromres.2020.04.024

11. Rotzinger DC, Beigelman-Aubry C, von Garnier C, Qanadli SD. Pulmonary embolism in patients with COVID-19: Time to change the paradigm of computed tomography. Thromb Res 2020;190:5859. https://doi.org/10.1016/j.thromres.2020.04.011

12. Bajc M, Schümichen C, Grüning T, et al. EANM guideline for ventilation/perfusion single-photon emission computed tomography (SPECT) for diagnosis of pulmonary embolism and beyond. Eur J Nucl Med Mol Imaging 2019;46:2429-2451. https://doi.org/10.1007/s00259-019-04450-0

13. Lu Y, Macapinlac HA. Perfusion SPECT/CT to diagnose pulmonary embolism during COVID-19 pu Y, Macapinlac HA. Perfusion SPECT/CT to diagnose pulmonary embolism during COVID-19
pandemic. Eur J Nucl Med Mol Imaging 2020;47:2064-2065. https://doi.org/10.1007/s00259-020pandemic. Eur J Nucl Med Mol Imaging 2020;47:2064-2065. https://doi.org/10.1007/s00259-020-
04851-6

14. Mahaletchumy T, Muhamad M, Umar S. Diagnosis of pulmonary embolism: A comparison between ventilation/perfusion SPECT/CT and perfusion-only SPECT/CT. Med J Malaysia 2020;75(5):490493

15. Vitali C, Minniti A, Caporali R, del Papa N. Occurrence of pulmonary embolism in a patient with mild clinical expression of COVID-19. Thromb Research 2020;192:21-22. https://doi.org/10.1016/j. thromres.2020.05.002

16. Vechi HT, Maia LR, do Monte Alves M. Late acute pulmonary embolism after mild coronavirus disease 2019 (COVID-19): A case series. Rev Inst Med Trop Sao Paulo 2020;62:63. https://doi. org/10.1590/s1678-9946202062063

17. Joseph J, Roberts J, Weaver C, Anderson J, Wong M. Patients with mild COVID-19 symptoms and coincident pulmonary embolism: A case series. Clin Pract Cases Emerg Med 2020;4(3):295-298. https://doi.org/10.5811/cpcem.2020.7.48254

18. Davis K. COVID-19 pneumonia with back pain: Presentation of an acute pulmonary embolism Davis K. COVID-19 pneumonia with back pain: Presentation of an acute pulmonary embolism
associated with novel coronavirus infection in an outpatient setting. Clin Case Rep 2020;8(12):25142517. https://doi.org/10.1002/ccr3.3208

19. Moradi F, Morris TA, Hoh CK. Perfusion scintigraphy in diagnosis and management of thromboembolic pulmonary hypertension. Radiographics 2019;39(1):169-185. https://doi. org/10.1148/rg.201918007

Accepted 17 May 2021. 